\title{
AUTOANTIBODIES TO THE INSULIN- AND IGF1-RECEPTOR IN HUMAN SERA
}

\author{
Welsink T\#,1,2 Schwiebert $C^{\#, 1,3}$, Minich WB1, Schomburg L1 \\ \# These authors contributed equally to this work
}

${ }^{1}$ Institute for Experimental Endocrinology, Charité, Berlin, Germany, ${ }^{2} I \mathrm{Cl}$ Immunochemical Intelligence GmbH, Berlin, Germany, ${ }^{3}$ InVivo BioTech Services GmbH, Hennigsdorf, Germany

\section{Introduction}

Autoantibodies $(\mathrm{A} A \mathrm{~B})$ are characteristic of autoimmune diseases, but may also be found in apparently healthy individuals and precede pathological symptoms. We have recently reported on $\mathrm{AAB}$ against the IGF1-receptor (IGF1R-aAB) in control subjects and patients with Graves' disease (1). The isolated $\mathrm{A} A \mathrm{~B}$ were able to immunoprecipitate the recombinant autoantigen and antagonized IGF1 signaling in vitro. Given the structural similarities between IGF1R and insulin receptor (IR), we decided to establish a diagnostic test for IR-aAB similar to our IGF1R aAB assay. IR-aAB are of endocrine importance as they may cause insulin resistance type $B$. Up to now, there is no such test available for routine testing, despite some radioactive and cumbersome assays (2). There are thus no data on the prevalence of $\mathrm{aAB}$ to the insulin and IGF-1 receptor in the normal population or in diseased individuals. For this reason, we decided to establish a suitable non-radioactive test system for the detection and quantification of these $a A B$ and to evaluate the prevalence of these $a A B$ in human sera.

\section{Objective}

To compare the prevalence and potential cross-reactivity of IGF1R-aAB and IR-aAB.

\section{Material and Methods}

Recombinant variants of both receptors were stably expressed in HEK293 cells and used to establish two diagnostic aAB assays, one of which having been described recently (1). Therefore firefly luciferase was fused $C$ terminal to the IR or IGF1R respectively.

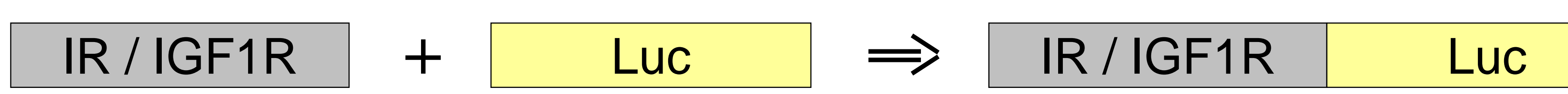

Figure 1: Stable expression of recombinant IR /IGF1R and Luciferase as fusion protein

Cell extracts of IR- or IGF1R-Luc fusion proteins were prepared and used for incubation with patient serum. If serum contained $A A B$ to the receptor fusion protein (containing the endogenous autoantigen IR or IGF1R), the antigen-antibody complex was bound and precipitated by a protein-A matrix (PA). Unbound components were removed and nonspecific binding was reduced by repeated washing and centrifugation. The precipitate obtained was detected by a CT luminometer using a luciferase-specific substrate. The relative light units $(R L U)$ obtained were directly proportional to the concentration of $\mathrm{aAB}$ in the human serum sample.
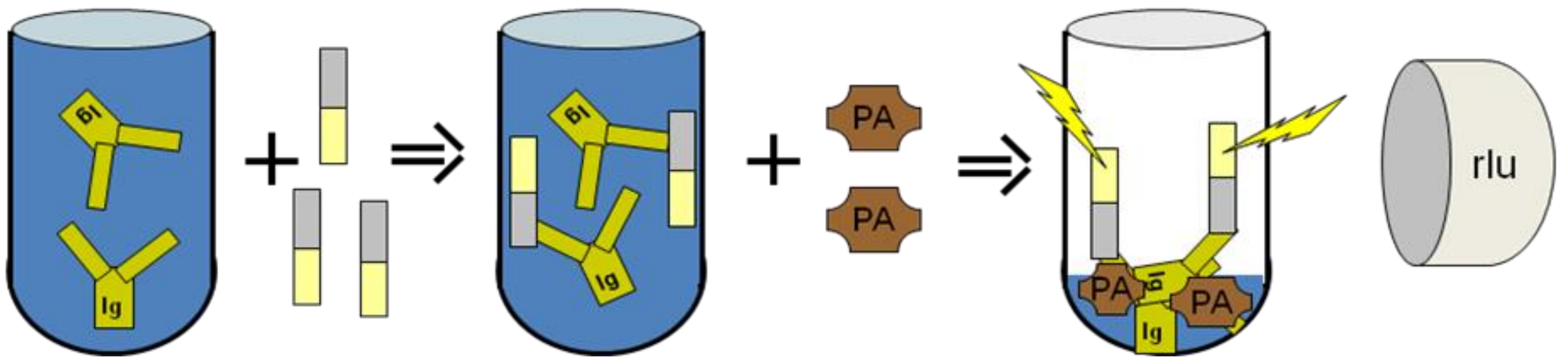

Figure 2: Schematic depiction of the diagnostic $A A B$ assay system using IR- or IGF1Rluciferase fusion proteins for precipitation.

Table 1A (right): Assay characteristics from IR-aAB and IGF1R-aAB precipitation assays.

Table 1B (below): Coefficients of variation for IR-aAB and IGF1R-aAB precipitation assay were determined by repeated assay were determined by repeat
measurements of the same samples.

\begin{tabular}{|c|c|c|c|c|}
\hline \multicolumn{3}{|c|}{ isurements of the same samples. } & \multicolumn{2}{|l|}{ ter. } \\
\hline CV & IR-aAB & IGF1R-aAB & $\begin{array}{l}\rightarrow \text { serum + extract } \\
\rightarrow+\text { protein A suspension }\end{array}$ & $\begin{array}{c}\text { overnight } \\
1 \mathrm{~h}\end{array}$ \\
\hline Inter & $18.4 \%$ & $13.6 \%$ & $\rightarrow$ washing & $3 \times 1 \mathrm{ml}$ \\
\hline Intra & $9.1 \%$ & $9.9 \%$ & $\rightarrow$ detection & $1 \times 10 \mathrm{sec}$ \\
\hline
\end{tabular}

A total number of 207 samples from a commercial supplier were analyzed for IGF1R-aAB and IR-aAB. Ethical approval and a written consent from each blood donor had been maintained before starting the analyses.

\section{Results}

A comparable number of sera turned out to be positive for IGF1R-aAB and IR-aAB, respectively, with a prevalence of approx. $5 \%$ each. A fraction of positive samples turned out to react with both the IGF1R and the IR. This result either indicates the presence of multiple specific-aAB isoforms or of one type of cross-reacting $\mathrm{A} A \mathrm{~B}$ in a given human serum sample.

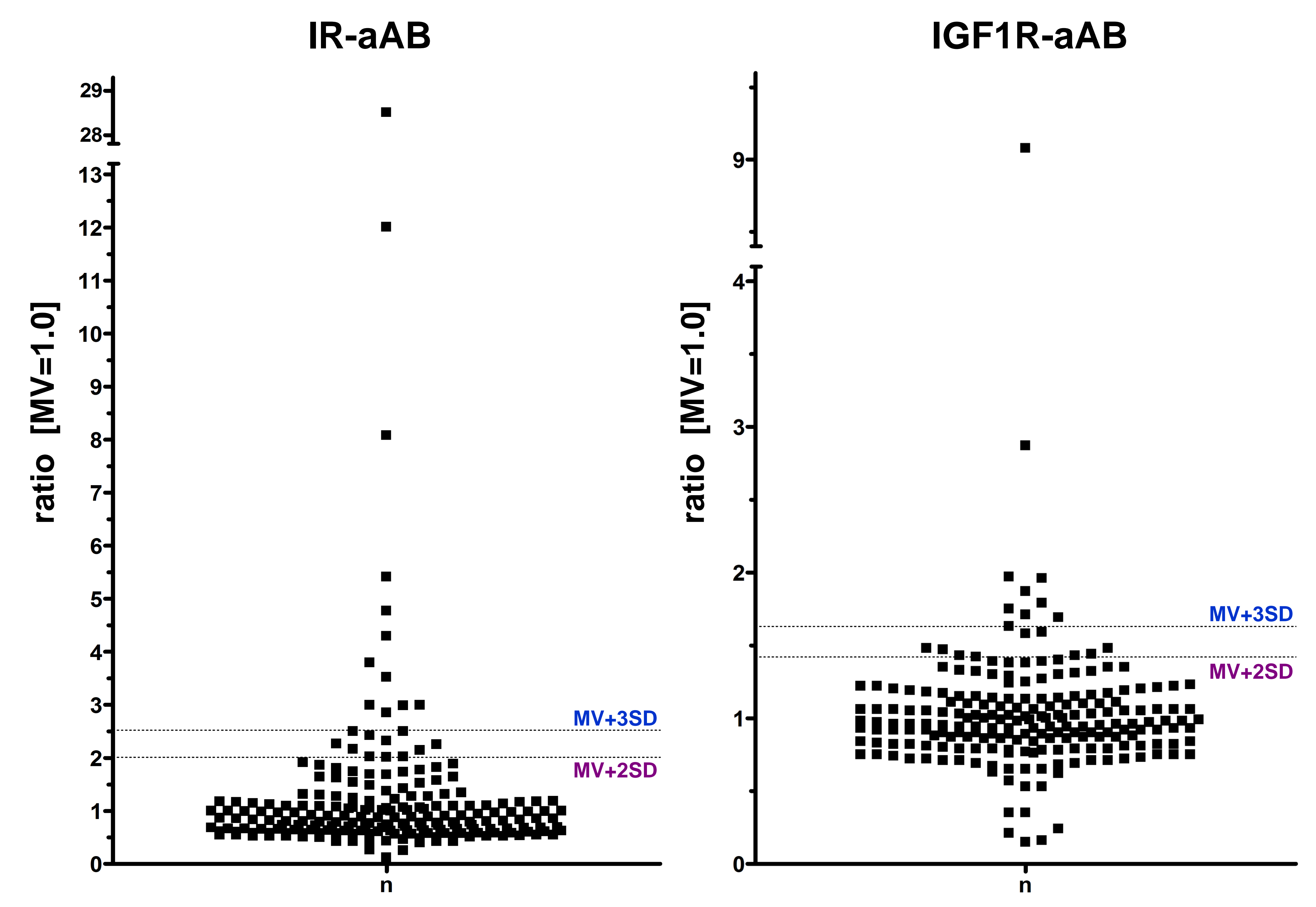

Diagram 1: Determination of IR- and IGF1R aAB in 207 serum samples using an recombinan autoantigen specific precipitation assay. Mean value (MV) and SD were calculated after excluding the upper and lower $5 \%$ of values. To calculate relative concentrations the MV was set to 1.0 . Values above MV+2SD were classified as slightly positive, values above MV+3SD as clearly positive.

\begin{tabular}{|c|c|c|c|c|}
\hline Assay & \multicolumn{2}{|c|}{ IR-aAB } & \multicolumn{2}{c|}{ IGF1R-aAB } \\
\hline Unit & RLU & MV $=\mathbf{1 . 0}$ & RLU & MV $=\mathbf{1 . 0}$ \\
\hline MV & 4680 & 1 & 12630 & 1 \\
\hline SD & 2366 & 0,51 & 2653 & 0,21 \\
\hline MV+2SD & 9412 & 2,01 & 17936 & 1,42 \\
\hline MV +3SD & 11779 & 2,52 & 20589 & 1,63 \\
\hline \begin{tabular}{|c|c|c|c|} 
Positive \\
sera
\end{tabular} & $\begin{array}{c}\text { IR-aAB } \\
\text { [n] }\end{array}$ & $\begin{array}{c}\text { Prevalence } \\
{[\%]}\end{array}$ & $\begin{array}{c}\text { IGF1R-aAB } \\
\text { [n] }\end{array}$ & $\begin{array}{c}\text { Prevalence } \\
{[\%]}\end{array}$ \\
\hline$>$ MV+2SD & 23 & 11,1 & 18 & 8,7 \\
\hline$>$ MV+3SD & 12 & 5,8 & 10 & 4,8 \\
\hline
\end{tabular}

Table 2A: Key data of IR- and IGF1R-aAB assay for 207 serum samples. Both the direct RLU values and the calculated ratios with respect to the mean value (MV) are given with threshold values for 2 or $3 S D$. Table 2B: Prevalence of aAB to IR and IGF1R in 207 serum samples. Both the absolute number and percentage of $a A B$ positive samples are indicated.

\begin{tabular}{|c|c|c|c|c|}
\hline \multirow{2}{*}{$\begin{array}{c}\text { Criteria } \\
\text { for pos. }\end{array}$} & \multicolumn{2}{|c|}{ IR-aAB } & \multicolumn{2}{c|}{ IGF1R-aAB } \\
\cline { 2 - 5 } & $>\mathrm{MV}+2 \mathrm{SD}$ & $>\mathrm{MV}+3 \mathrm{SD}$ & $>\mathrm{MV}+2 \mathrm{SD}$ & $>\mathrm{MV}+3 \mathrm{SD}$ \\
\hline Correlation & $35 \%$ & $42 \%$ & $45 \%$ & $50 \%$ \\
\hline
\end{tabular}
Table 2C: Correlation of pos. samples. Percentages indicate number of sera being positive for both $\mathrm{AAB}$ tested.
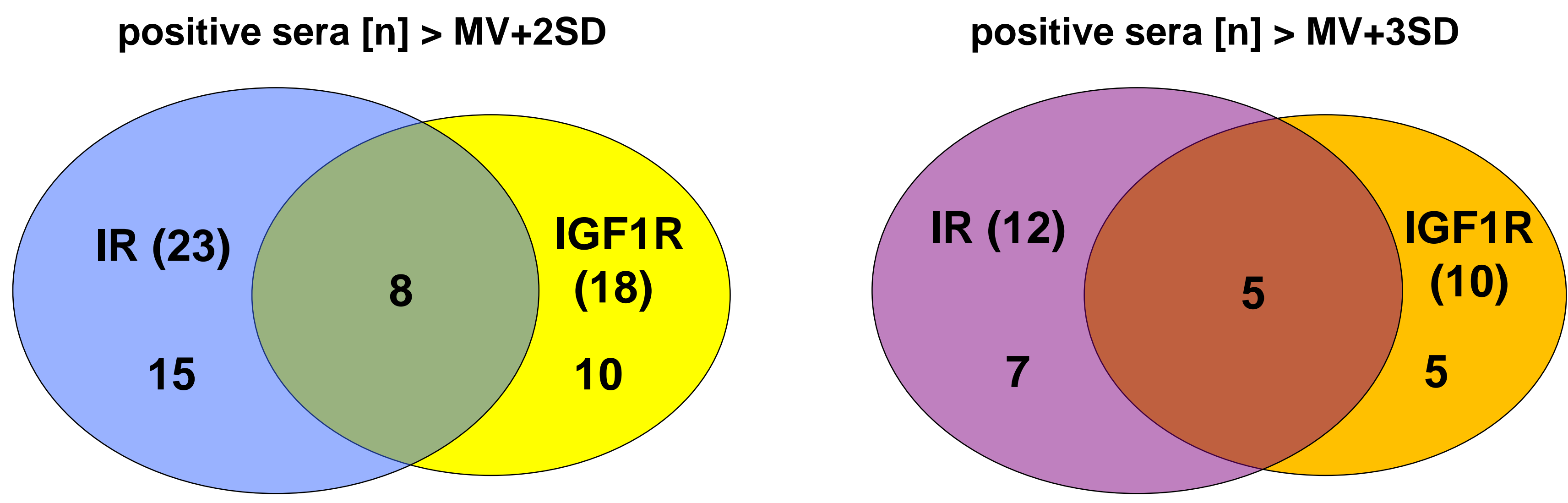

Figure 3: Correlation of samples being simultaneous positive for IR- and IGF1R-autoimmunity as defined by a stringent $(M V+3 S D)$ and less stringent $(M V+2 S D)$ criterion for aAB-positivity.

\section{Conclusions}

Our studies demonstrate a prevalence of around $5 \%$ for aAB to IGF1R and $\mathrm{IR}$ in the analyzed sera. We found an unexpected high correlation of samples being simultaneously positive for IGF1R- and IR-aAB. These findings may be of clinical relevance for the current diabetes epidemic, but further analyzes are needed to test this hypothesis with patient sera.

\section{Acknowledgements}

Supported by German Federal Ministry of Economics and Technology (BMWi), project KF2775801AJ0 within the ZIM program, and by GraKo 1208/2 program of the DFG. 